\title{
MODELS FOR SHORT-RANGE WAKEFIELDS IN PLANAR STRUCTURES*
}

\author{
A.V. Smirnov, D. Yu, DULY Research Inc., Ranch Palos Verdes, CA, 90275
}

\begin{abstract}
Diffraction models are developed for high-frequency radiation losses calculation in rectangular iris-loaded structures. Single-cell and multi-cell models extending Lawson and Sessler-Vainshtein approaches are applied for circular, infinitely wide planar and 'muffin-tin' geometry. For 2.5D muffin-tin structure a special semi-analytical frequency domain model is built to compare the loss calculations between diffraction model and matched field technique.
\end{abstract}

\section{INTRODUCTION}

Nowadays planar structures draw growing attention to application in future linear colliders based on mm-wave structures [1]. To characterize the wakefields induced in non-circular (and particularly in planar) structures, corresponding models must be developed.

Well-known diffraction models for a single cavity (Lawson model [2,3]) and for periodic structures (SesslerVainshtein model in [4]) exploit structure symmetry and uniformity of incident energy flow. For planar structures, this assumption is no longer valid especially when beam geometry is not similar to aperture geometry. To generalize the diffraction model approach we apply the Green function technique along with 'image' field concept for monopole wakefield. Frequency-domain model is developed as well and applied for muffin-tin structure.

\section{EXTENDED DIFFRACTION MODELS}

We treat the diffraction as though caused by image fields induced by the beam on the iris edge. Neglecting iris thickness, the incident image fields can be defined in frequency domain as follows:

$$
\Delta_{\perp} \tilde{E}_{z}^{(i)}-\frac{k^{2}}{\beta^{2} \gamma^{2}} \tilde{E}_{z}^{(i)}=0,\left.\tilde{\vec{E}}_{\perp}^{(i)}\right|_{\text {iris edge }}=-\left.\tilde{\vec{E}}_{\perp}^{(s p)}\right|_{\text {iris edge }},
$$

where $\gamma=1 / \sqrt{1-\beta^{2}}$ is the relativistic factor, $\tilde{\vec{E}}^{(i)}$ and $\tilde{\vec{E}}^{(s p)}$ are the Fourier transforms of the image field and the space charge field in a free space correspondingly.

The fields diffracted by single iris can be obtained from wave parabolic equation valid at $k r^{2}>>, \sqrt{k z}>1$ :

$$
\partial \tilde{W} / \partial z=(i / 2 k) \Delta_{\perp} \tilde{W}
$$

where $\tilde{\Phi}(\vec{r})=\tilde{W}\left(\vec{r}_{\perp}, z\right) e^{i k z}$ is field potential function; $\tilde{W}\left(\vec{r}_{\perp}, z\right)$ is a slow varying amplitude function; and $\tilde{W}\left(\vec{r}_{\perp}, 0\right)$ corresponds to the image fields. Diffraction radiation losses in a single cell composed of two irises spaced by distance $\Lambda$ can be found using the Poynting vector and Babinet's principle:

$$
\frac{d U}{d \omega}=\frac{2}{\pi} \int_{S_{\perp}}^{\infty} d S\left(\tilde{\vec{E}}\left(\vec{r}_{\perp}, \Lambda\right) \times \tilde{\vec{H}}^{*}\left(\vec{r}_{\perp}, \Lambda\right)\right) \vec{e}_{z} .
$$

For a long periodic structure $\left(N_{p e r}>2 N_{f}\right.$, see $\left.[5,6]\right)$ we apply an extended formulation of the Sessler-Vainshtein approach [4]. The optical cavity modal loss factor $\alpha_{s}$ corresponds to a single pass of the diffracted wave excited by the incident ('image') field. Hence, the structure of the equivalent optical mode is defined by the 'image' field. Taking into account different field patterns for nonuniform (across the aperture) incident field we introduce generalized expression for high frequency losses in a periodic structure:

$$
U=\sum_{s} \frac{\alpha_{s} C_{s}}{2 \pi} \int_{\omega_{1}}^{\infty} d \omega \int_{\text {aperture }}^{\infty} d x d y\left(\tilde{\vec{E}}^{(i)}(x, y) \times \widetilde{\tilde{H}}^{(i)} *(x, y)\right) \vec{z}_{z},
$$

where $C_{s}$ is the coupling coefficient for the quasi-Eigen optical mode $s$.

\section{SIMPLE GEOMETRIES}

The first example is a circular aperture of radius $a$. From (1) we can express transverse 'image' fields induced by the point charge $q$ as follows:

$\tilde{E}_{r}^{(i)}=c \widetilde{B}_{\theta}^{(i)}=\frac{-q}{2 \pi \varepsilon_{o}} \frac{k}{\beta \gamma} I_{1}\left(\frac{k r}{\beta \gamma}\right) K_{1}\left(\frac{k a}{\beta \gamma}\right) / I_{1}\left(\frac{k a}{\beta \gamma}\right) \approx \frac{-q}{2 \pi \varepsilon_{o}} \frac{r}{a^{2}}$. Assuming $\tilde{E}_{r}^{(i)}(r)=\tilde{W}(r, 0), \gamma>>k a$ we find from (2) the diffracted field using 1D Green function: $\widetilde{E}_{r}(r, z)=\tilde{W}=$

$\frac{-q a^{-2}}{2 \pi \varepsilon_{o}}\left[\sqrt{\frac{z}{2 \pi k}}\left(e^{i \frac{k r^{2}}{2 z}}-e^{i \frac{k(r-a)^{2}}{2 z}}\right) e^{i \frac{\pi}{4}}+r\left(F\left(r \sqrt{\frac{k}{z}}\right)-F\left((r-a) \sqrt{\frac{k}{z}}\right)\right)\right]$,

where $F(\tau)=\left(e^{-i \pi / 4} / \sqrt{2 \pi}\right) \int_{-\infty}^{\tau} d x e^{i \pi x^{2} / 2}$ is Fresnel function.

Now we can calculate the spectral losses of a point charge with corresponding 1D integration (see Fig. 1): $d U / d \omega \approx 2\left(\varepsilon_{o} / \pi c\right) 2 \pi a \int_{a}^{r_{\max }} d r\left|\tilde{E}_{r}(r, \Lambda)\right|^{2}$.

For periodic circular structure only one radially polarized ('donut' shaped) mode $T M_{01}^{(r)}$ is dominant. Then (4) at $k a<<, \quad C_{s}=1 \quad$ yields: $d U / d \omega=\alpha_{o} q^{2} /\left(4(2 \pi)^{2} c \varepsilon_{o}\right)$, where $\alpha_{o}=8 j_{11}{ }^{2} \beta_{\eta}\left(M+\beta_{\eta}\right) /\left[\left(M+\beta_{\eta}\right)^{2}+\beta_{\eta}{ }^{2}\right]^{2}, \beta_{\eta}=0.824$,

* This work is supported by DOE SBIR No. . DE-FG03-96ER82213. 
$M=\sqrt{8 \pi N_{f}}, \quad j_{l l}=3.832, \quad$ and $N_{f}=\omega a^{2} / 2 \pi c \Lambda$. Note, previous models used $j_{01}=2.405$ for monopole mode whereas it correspond to asymmetric (dipole) modes.

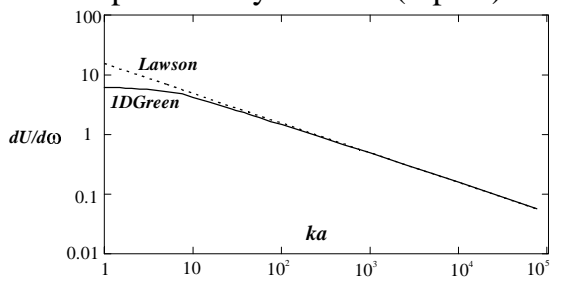

Figure 1: Spectral losses $\left[\mathrm{J} \cdot \mathrm{s} / \mathrm{C}^{2}\right]$ for the extended model (solid curve) and the Lawson formula [2,4] (dotted line). $a=0.3 \mathrm{~mm}, \Lambda=0.633 \mathrm{~mm}, \omega_{\min }=c / a=10^{12} \mathrm{c}^{-1}, \gamma>>k a$.

The second example is two symmetric infinitely wide comb arrays with a horizontal line charge moving between them. The 'image' field derived from (1) is: $\tilde{E}_{y}{ }^{(i)}(y)=\frac{-q^{\prime}}{2 \varepsilon_{o}} \operatorname{coth}\left(\frac{k b}{\beta \gamma}\right) \sinh \left(\frac{k y}{\beta \gamma}\right) \underset{\gamma \rightarrow \infty}{\longrightarrow} \frac{-q^{\prime}}{2 \varepsilon_{o}} \frac{y}{b}$,

where $q^{\prime}=d q / d x$ is the linear charge density, and $2 b$ is the vertical gap. Single cell spectral losses per unit length follow from $(2,3)$ at $k b<<\gamma$ (see Fig. 2, solid curve):

$$
\frac{d^{2} U}{d \omega d x}=\frac{q^{\prime 2} k / \Lambda}{2 \pi^{2} \varepsilon_{o} c} \int_{b}^{y_{\max }} d y\left|\int_{-b}^{b} \frac{y_{1}}{b} \exp \left(\frac{i k}{2 \Lambda}\left(y-y_{1}\right)^{2}\right) d y_{1}\right|^{2} \text {. }
$$

Straightforward application of the uniform plane wave approach [2] gives at $E_{y}=\widetilde{E}_{y}^{(i)}(b)$ (see Fig. 2, dotted line): $\frac{d^{2} U}{d \omega d x} \approx \frac{q^{\prime 2} 0.44}{2 \pi \varepsilon_{o} c} \sqrt{\frac{\pi \Lambda}{2 k}} \cosh ^{2}\left(\frac{k b}{\beta \gamma}\right)$.

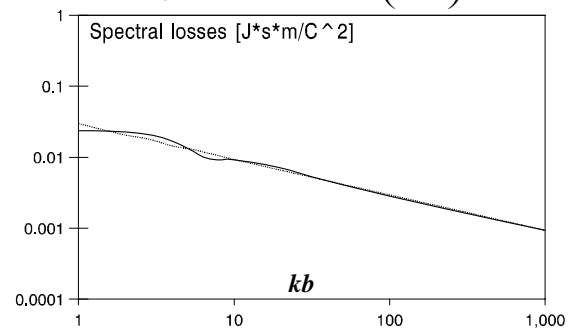

Figure 2: Spectral losses of line charge between two pairs of semi-infinite screens. Solid curve: extended approach; dotted line: Lawson (plane-wave) approach. $b=0.3 \mathrm{~mm}$, $\Lambda=0.633 \mathrm{~mm}, \omega_{\min }=c / b=10^{12} \mathrm{c}^{-1}, \gamma>>k b$.

For a periodic structure one can get from (4):

$$
\begin{aligned}
& \frac{d^{2} U}{d \omega d x}=\frac{q^{\prime 2} b \alpha_{I I}}{24 \pi c \varepsilon_{o}}, \quad \text { where } \alpha_{I I}=\frac{2 \pi^{2} n^{2} \beta_{\eta}\left(M_{y}+\beta_{\eta}\right),}{\left[\left(M_{y}+\beta_{\eta}\right)^{2}+\beta_{\eta}{ }^{2}\right]^{2}}, \quad n=2, \\
& M_{y}=\sqrt{8 \pi N_{y}}, N_{y}=\omega b^{2} / 2 \pi c \Lambda \text {. }
\end{aligned}
$$

Simple comparison of modal loss-factors for wide planar and circular cases at $a=b$ gives: $\alpha_{I I} / \alpha_{o} \approx 0.672$.

\section{RECTANGULAR STRUCTURE}

We consider next a point charge passing a muffin-tin structure $[1,8,9]$. Within the framework of the diffraction model we assume thin irises at $|y| \geq b$ without imposing any boundary conditions at the cavity walls $x= \pm a$ or side opening walls $x= \pm d$. Rigorous solution of (1) found for this boundary problem is:

$$
\begin{gathered}
\text { for } \mathrm{X}^{2}<<1: \tilde{E}_{z}^{(i)}=-q I_{1}(k r / \beta \gamma) \sin \varphi /\left(\gamma \pi \varepsilon_{o} b\right), \\
\tilde{\vec{E}}_{\perp}^{(i)}=-\frac{q}{2 \pi \varepsilon_{o} b}\left(I_{0}\left(\frac{k r}{\beta \gamma}\right)+I_{2}\left(\frac{k r}{\beta \gamma}\right)\right) \sin \varphi\left(\begin{array}{c}
\cos \varphi \\
\sin \varphi
\end{array}\right), \\
\text { for } \mathrm{X}^{2}>>1: \quad \tilde{E}_{z}^{(i)}=i \sqrt{i / 2} I_{0}(k r / \beta \gamma) q k /\left(\varepsilon_{o} \beta \gamma^{2}\right), \\
\tilde{\vec{E}}_{\perp}^{(i)}=\frac{q}{\varepsilon_{o}} \frac{k}{\beta \gamma} i \sqrt{\frac{i}{2}} I_{1}\left(\frac{k r}{\beta \gamma}\right)\left(\begin{array}{c}
\cos \varphi \\
\sin \varphi
\end{array}\right) \text {, where } \mathrm{X}=\frac{k b}{\beta \gamma \sin \varphi},
\end{gathered}
$$$$
\varphi=\arctan (y / x), r=\sqrt{x^{2}+y^{2}} \text {. }
$$

To find single cell diffracted fields we must substitute (6) into 2D Green function corresponding to (2). However, it leads to numerical problems caused by diverging integration. One can find approximated closed form neglecting transverse diffusion of the diffracted fields at $(k b)^{2} \gg>1 \sqrt{N_{f}}>1$. From the analytical part of the examples above one can see that the diffracted fields are dominated by the terms with Fresnel function. For rectangular iris these approximations give rise to the following simplified relationship between the diffracted and incident fields:

$$
\left.\tilde{\vec{E}}_{\perp}(x, y, z)\right|_{z=\Lambda} \approx \tilde{\vec{E}}_{\perp}{ }^{(i)}(x, b) \cdot D\left((y-b) / b, N_{y}\right),
$$

where $D\left(\xi, N_{y}\right)=F\left((\xi+1) \sqrt{2 \pi N_{y}}\right)-F\left(\xi \sqrt{2 \pi N_{y}}\right)$.

In this way the Lawson approach results in simplified formula of radiation losses in a muffin-tin cell:

$$
\frac{d^{2} U}{\partial \omega \partial x} \approx \frac{4 b}{\pi}\left(\int_{0}^{\infty}\left|D\left(\xi, N_{y}\right)\right|^{2} d \xi\right)\left(\widetilde{\tilde{E}}^{(i)}(x, b) \times \tilde{\vec{H}}^{(i)} *(x, b)\right) \vec{e}_{z}(8)
$$

Substitution (7) into (8) gives the following expression valid at $1<<(k b)^{2}<<\gamma$ :

$$
d U / d \omega \approx 0.00755 q^{2} \sqrt{\lambda \Lambda} \arctan A /\left(b \varepsilon_{o} c\right),
$$

where $A=a / b$ is the aspect ratio for the iris aperture. One can see, point charge energy losses in a single muffin-tin cell are defined by a factor of $R \cong 0.665 \operatorname{arctg} A$ with respect to circular structure of the same aperture radius.
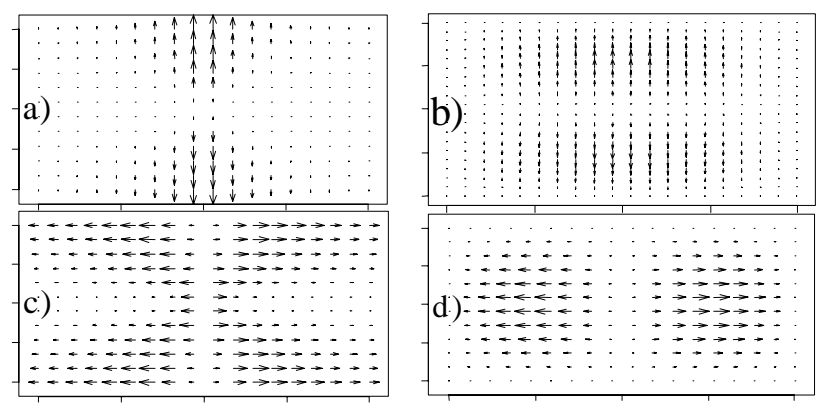

Figure 3: Field patterns for incident field vertical $\tilde{E}_{y}^{(i)} \vec{e}_{y}$ (a), horizontal $\tilde{E}_{x}^{(i)} \vec{e}_{x}$ (c) components and corresponding rectangular optical cavity modes $T E_{12}^{(y)}$ (b) and $T E_{21}^{(x)}$ (d).

In the model of periodic rectangular structure we deal with excitation of two independent, quasi-Eigen modes of 
an optical resonator: $T E_{21}^{(x)}$ and $T E_{12}^{(y)}$. Both modes are the lowest and have the same symmetry as the incident fields $\widetilde{E}_{x}^{(i)} \vec{e}_{x}+\widetilde{E}_{y}^{(i)} \vec{e}_{y}$ (see Fig. 3).

Using (6) we can determine the coupling coefficients $C_{l, 2}$ for the optical modes as follows: $C_{1,2}=\int_{-a}^{a} \int_{-b}^{b} d x d y \tilde{E}_{x, y}^{(i)} \tilde{H}_{y, x}^{(i)} * / \int_{-a}^{a} \int_{-b}^{b} d x d y\left(\tilde{\vec{E}}^{(i)} \times \tilde{\vec{H}}^{(i)} *\right) \vec{e}_{z}$, $\frac{C_{1}}{C_{2}}=\frac{-A+\left(1-A^{2}\right) \operatorname{arctg} A+\pi / 2}{3 A-\left(3+A^{2}\right) \operatorname{arctg} A^{-1}+\pi / 2}, \quad C_{1}+C_{2}=1$.

The attenuation factors for the optical modes $T E_{21}^{(x)}$ and $T E_{12}^{(y)}$ can be found from Vainshtein theory [7]: $\alpha_{1,2}=\frac{2 \pi^{2}}{\beta_{\eta}^{2}}\left\{\frac{m_{1,2}^{2}\left(M_{x} / \beta_{\eta}+1\right)}{\left[\left(M_{x} / \beta_{\eta}+1\right)^{2}+1\right]^{2}}+\frac{n_{1,2}{ }^{2}\left(M_{y} / \beta_{\eta}+1\right)}{\left[\left(M_{y} / \beta_{\eta}+1\right)^{2}+1\right]^{2}}\right\}$, where $\quad m_{1,2}=(2,1) ; \quad n_{1,2}=(1,2) ; \quad M_{x, y}=\sqrt{8 \pi N_{x, y}}$, $N_{x}=\omega d^{2} / 2 \pi c \Lambda$.

Finally we obtain the spectral losses for muffin-tin periodic structure in the following form: $\frac{d U}{d \omega}=\frac{q^{2} \pi^{-3}}{4 \varepsilon_{o} c}\left[A+\left(1+A^{2}\right) \operatorname{arctg} A-\frac{\pi}{2} A^{2}\right] \sum_{s=1,2} \alpha_{s} C_{s}$.

For an infinitely wide periodic structure $(a, d)>>b$ we have $C_{1}=C_{2}=1 / 2$ and the losses are of factor of $R=5 \pi^{2} / 4 j_{11}{ }^{2} \approx 0.84$ with respect to cylindrical periodic structure having radius $b$. Iris aspect ratio influence on the relative radiation losses is shown in Fig.4.

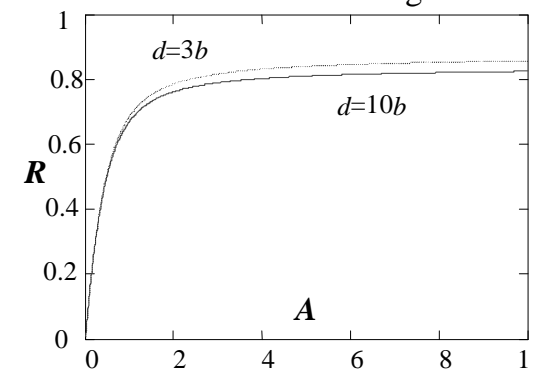

Figure 4: Radiation losses with respect to circular structure $(r=b)$ versus iris aperture aspect ratio $A=a / b$.

\section{FREQUENCY DOMAIN SIMULATIONS}

A semi-analytic model is built to calculate synchronous monopole modes. It is based on matching field technique applied to a $2.5 \mathrm{D}$ muffin-tin structure without side openings (i.e. $d=a$ ). The basic source relationships for eigenmodes in a muffin-tin structure are given, e.g., in refs. [8,9]. We included modes having up to $M=7$ variations along the horizontal axis; higher modes at $M \geq 9$ have negligible contribution. In Fig. 5 we present the results of the calculated energy loss spectrum obtained by summing modal loss factors with averaging over frequency bins of constant width. The superposed curve in this figure is an analytical calculation given by (12). Integrated losses over finite frequency range of variable length are given in Fig. 6 for both models. Minimal frequency of integration corresponds to the second monopole mode at $k b=1.64, f_{\text {min }}=\omega_{\text {min }} / 2 \pi=259.4 \mathrm{GHz}$ (the first is fundamental mode $k b=0.785$ ).

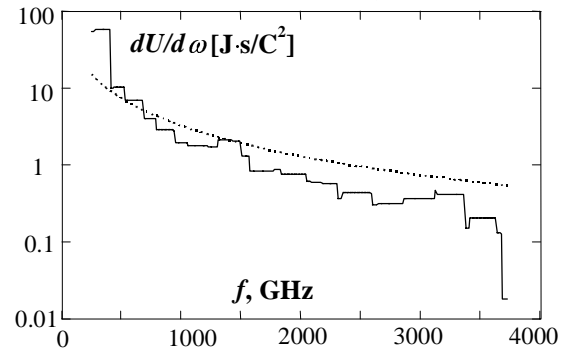

Figure 5: Spectral losses for matched fields frequency domain model (solid curve) and the extended SesslerVainshtein model (dotted curve) for 2.5D muffin-tin structure. $b=0.3 \mathrm{~mm}$, aperture cross-section $6 b \times 2 b$, $\Lambda=0.633 \mathrm{~mm}, a=d=0.9 \mathrm{~mm}$.

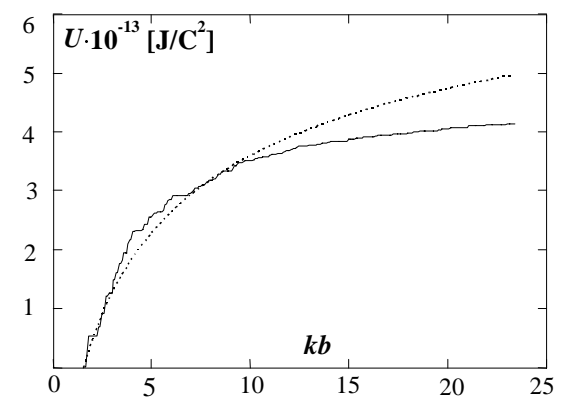

Figure 6: Energy losses integrated over finite frequency range versus $\mathrm{kb}$ for $2.5 \mathrm{D}$ structure (see Fig. 5). Solid curve: matched field technique for a frequency domain model; dotted curve: the Sessler-Vainshtein model.

\section{ACKNOWLEDGEMENTS}

The authors are very appreciative of Prof. A. W. Chao and Dr. S. A. Kheifets for useful discussions and essential remarks.

\section{REFERENCES}

[1] D. Yu, S. Ben-Menahem, P. Wilson, R. Miller, R. Ruth, A. Nassiri, in AIP Conf. Proc., AIP 335, edited by P. Schoessow, (1994) 800.

[2] J.D. Lawson, Particle Accelerators, v. 25 (1990) 107.

[3] K. Bane and M. Sands, SLAC-PUB-4441 (1987).

[4] E. Keil, Nucl. Instr. and Meth., NIMA100 (1972) 419.

[5] S. A. Heifets, S. A. Kheifets, SLAC-PUB-4625 (1988).

[6] A.V. Smirnov, in Proc. of Particle Accelerator Conference, (PAC95), Dallas, Texas, (1999) 3615.

[7] L.A. Vainshtein, Open Resonators and Open Waveguides, Izdatelstvo "Sovetskoe Radio," Moscow, 1966 (in Russian).

[8] M. Filtz, in Proc. of European Particle Accelerator Conference (EPAC94), London, 1994, p. 1271.

[9] M. Filtz, in Proc. of Particle Accelerator Conference (PAC'95), Dallas, Texas, May, 1995, p. 2373. 